\title{
Advances in depression research: special issue, 2020, with three research articles by Paul Greengard
}

\author{
Julio Licinio' ${ }^{1}$ Ma-Li Wong ${ }^{1}$
}

C Springer Nature Limited 2020

There has been stunning progress in depression research, which is reflected in the pages of this special issue of Molecular Psychiatry. Our last special issue was dedicated to stress and behavior; that is one of the key biological pathways leading to depression. Building on our previous special issue, this issue directly addresses depression.

This special depression issue has three articles by the late Nobel laureate Paul Greengard. In the first of those papers, Sagi et al. focus on parvalbumin interneurons, which are a major class of GABAergic neurons, essential for hippocampal dentate gyrus (DG) function [1]. They demonstrated, in mice, that hippocampal parvalbumin interneurons express functionally silent serotonin 5A receptors, which translocate to the cell membrane and become active upon chronic, but not acute, treatment with a selective serotonin reuptake inhibitor (SSRI). Activation of these serotonergic receptors in these neurons initiates a signaling cascade through which Gi-protein reduces cAMP levels and attenuates protein kinase $\mathrm{A}$ and protein phosphatase $2 \mathrm{~A}$ activities. This results in increased phosphorylation and inhibition of Kv3.1 $\beta$ channels, and thereby reduces the firing of the parvalbumin neurons. Through the loss of this signaling pathway in these neurons, conditional deletion of the serotonin 5A receptor leads to the loss of the physiological and behavioral responses to chronic antidepressants. This illustrates the mechanisms by which serotonergic signaling in DG mediates the effects of antidepressant treatment. The second paper addresses the biological underpinnings of the delayed onset of action of antidepressants, a topic of perennial interest in depression research. The elucidation of those mechanisms might herald the onset of newer, faster acting antidepressants. Oh et al.

Julio Licinio

juliolicinio@gmail.com

1 State University of New York, Upstate Medical University, Syracuse, NY 13210, USA propose that this therapeutic delay suggests slow adaptive changes in multiple neuronal subtypes and their neural circuits over prolonged periods of drug treatment [2]. They showed that neuronal activity of hippocampal mossy cells, which are excitatory neurons in the dentate hilus that regulate dentate gyrus activity and function, is enhanced by chronic, but not acute, SSRI administration. Their data established that mossy cells play a crucial role in mediating the effects of chronic antidepressant medication, and their results indicate that compounds that target mossy cell activity would be attractive candidates for the development of new antidepressant medications. In the third paper coauthored by Paul Greengard in this issue, Shuto et al. demonstrated that dopamine D1 receptors in the DG act as a pivotal mediator of antidepressant actions in mice and indicate that stimulation of D1 receptors in the dentate gyrus is a potential adjunctive approach to improve therapeutic efficacy of SSRI antidepressants [3].

The work by Greengard and collaborators is accompanied by other outstanding papers. Teissier et al. examined changes induced by the stress of chronic maternal separation during the first 2 weeks of postnatal life [4]. They showed that unbiased mRNA expression profiling in the medial PFC (mPFC) of maternally separated (MS) pups identified an increased expression of myelin-related genes and a decreased expression of immediate early genes. Bidirectional manipulation of neuron excitability in the mPFC during the P2-P14 period had long lasting effects on adult emotional behaviors and on temporal object recognition. These results identify neuronal activity as a critical target of early-life stress and demonstrate its function in controlling both postnatal oligodendrogenesis and adult mPFC-related behaviors.

Most papers in this issue pursue new mechanistic targets with potential impact on depression biology and therapeutics. The paper by Zhang et al. explores the potential role of circRNAs in major depressive disorder (MDD), showing that circular RNA DYM (circDYM) levels were decreased both in the peripheral blood of patients with 
MDD and in two depressive-like mouse models. Importantly, restoration of circDYM expression significantly attenuated depressive-like behavior and inhibited microglial activation induced by CUS or LPS treatment. Of note, further examination indicated that circDYM functions as an endogenous microRNA-9 (miR-9) sponge to inhibit miR-9 activity, which results in a downstream increase of targetHECT domain E3 ubiquitin protein ligase 1 (HECTD1) expression, an increase of HSP90 ubiquitination, and a consequent decrease of microglial activation [5]. They conclude that circDYM may be a novel therapeutic target for depression. This research contributes to an emerging and exciting body of work showing that circRNAs can regulate gene expression by influencing the transcription, the mRNA turnover, and translation by sponging RNA-binding proteins and microRNAs [6].

An additional new antidepressant target is pursued by Tan et al. who studied a promising novel pharmaceutical target, the KCNQ-type potassium channel, which may exert a therapeutic effect via functional changes within the brain reward system, including the ventral striatum [7]. They assessed the effects of the KCNQ channel opener ezogabine (also known as retigabine) on reward circuitry and clinical symptoms in patients with MDD. Their findings highlight the KCNQ-type potassium channel as a promising target for future drug discovery efforts in mood disorders.

Work by Kin et al. explored the effects of cell encapsulation on the survival rate of grafted cells and showed that encapsulation enhanced the antidepressant effects of mesenchymal stem cells (MSCs) by attenuating depressivelike behavior of Wistar Kyoto (WKY) rats, an animal model of treatment-resistant depression [8]. They put forward the concept that implantation of encapsulated MSCs into the lateral ventricle exerted antidepressant effects acting via neurogenic pathways, supporting their potential therapeutic role in the treatment of MDD.

It is known that most existing antidepressants block monoamine reuptake by high-affinity transporters in the brain and have slow onset of action and treatment resistance. Orrico-Sanchez et al. reported the development of a prodrug targeting organic cation transporters (OCT), which are atypical monoamine transporters recently implicated in the regulation of mood [9]. They designed a selective OCT2 blocker, H2-cyanome, which was modified to increase brain penetration. Prolonged administration of $\mathrm{H} 2$-cyanome in mice induced positive effects on depressive-like behaviors, including anhedonia, anxiety, social withdrawal, and memory impairment. The authors provide proof-of-concept of antidepressant efficacy of an OCT blocker, and a mechanistic framework for the development of new classes of antidepressants.

Han et al. evaluated the antidepressant effects of immunization with altered peptide ligands of myelin basic protein
(MBP)-MBP87-99[A91, A96], MBP87-99[A91], and MBP87-99[R91, A96] in different models of depression and examined the mechanism by which these peptides protect against stress-induced depression [10]. Their findings indicated that immunization with altered MBP peptide produces prolonged antidepressant-like effects in rats, and the behavioral response is mediated by inflammatory factors (particularly interleukin-6), and p11 signaling in the prelimbic cortex.

Immune mediators are also considered in a study of pharmacological trials. Wittenberg et al. examined the effects of immunomodulatory drugs on depressive symptoms through a mega-analysis of randomized, placebocontrolled clinical trials in inflammatory disorders [11]. Their findings are many and can be found in their paper. Briefly, they conclude that novel immune-therapeutics can produce antidepressant effects in depressed patients with primary inflammatory disorders that are not entirely explained by treatment-related changes in physical health. The role of inflammatory mediators is also explored by other papers in this issue. In a longitudinal study of urban adults, Beydoun et al. showed that systemic inflammation is associated with depressive symptoms differentially by sex and race [12]. Felger at al. collected plasma and cerebrospinal fluid (CSF) from unmedicated patients with MDD [13]. Plasma C-reactive protein (CRP) was correlated with multiple plasma inflammatory markers, and a strong correlation was found between plasma and CSF CRP. CSF CRP in turn correlated with CSF cytokine receptors/ antagonists. Clusters of CSF inflammatory markers were associated with high plasma CRP and correlated with depressive symptom severity. They concluded that CRP appears to be a peripheral biomarker that reflects peripheral and central inflammation and seems well-suited for guiding immunotherapies in patients with MDD.

The role of methylation in the genetics of complex disorders is a topic of great interest. We have two papers in this issue on methylation in MDD. Clark et al. associated blood DNA methylation profiles from MDD patients at baseline with MDD status 6 years later [14]. A resampling approach showed a highly significant association between methylation profiles in blood at baseline and future disease status. Top MWAS results were enriched specific pathways, overlapped with genes found in genome wide association studies (GWAS) of MDD disease status, autoimmune disease and inflammation. The major themes emerging were cellular responses to stress and signaling mechanisms linked to immune cell migration and inflammation. This suggests that an immune signature of treatment-resistant depression is already present at baseline. The authors propose that methylation profiles in blood may present a promising avenue to support clinical decision making by providing empirical information about the likelihood MDD is chronic 
or will recur in the future. In a pioneering paper, Aberg et al. presented the first large-scale methylome-wide association studies (MWAS) for MDD to identify and replicate methylated loci associated with MDD that are involved in biological functions of likely importance to MDD etiology [15].

It is expected that not every patient will respond to every treatment. Given the high numbers of existing treatments and MDD patients, predicting individualized treatment response is critically important. Wei et al. provided novel pharmacogenomic evidence to support the role of HTR7 in association with antidepressant response [16].

The fifteen research articles in this special issue on depression highlight the advances of this field, with immense progress achieved in the elucidation of biological mechanisms and pathways and the exploration of exciting new therapeutic targets. We are particularly honored to have three papers here that are authored by the late Paul Greengard, representing some of his most recent work. Further work from Greengard's lab will be coming out soon in a future issue of Molecular Psychiatry. In that exciting article, Chottekalapanda et al. put forth a sequential molecular network to track the antidepressant response and provide a new avenue that could be used to accelerate or potentiate antidepressant responses by triggering neuroplasticity [17]. As we go along, Molecular Psychiatry will continue to publish the most exciting advances in depression research with the aim of expanding the frontiers of knowledge and disseminating robust new strategies for MDD diagnosis and treatment.

\section{Compliance with ethical standards}

Conflict of interest The authors declare that they have no conflict of interest.

Publisher's note Springer Nature remains neutral with regard to jurisdictional claims in published maps and institutional affiliations.

\section{References}

1. Sagi Y, et al. Emergence of 5-HT5A signaling in parvalbumin neurons mediates delayed antidepressant action. Mol Psychiatry. https://doi.org/10.1038/s41380-019-0379-3 (2019).

2. Oh SJ, et al. Hippocampal mossy cell involvement in behavioral and neurogenic responses to chronic antidepressant treatment. Mol Psychiatry. https://doi.org/10.1038/s41380-019-0384-6 (2019).
3. Shuto $\mathrm{T}$, et al. Obligatory roles of dopamine $\mathrm{D} 1$ receptors in the dentate gyrus in antidepressant actions of a selective serotonin reuptake inhibitor, fluoxetine. Mol Psychiatry. https://doi.org/10. 1038/s41380-018-0316-x (2018).

4. Teissier A, et al. Early-life stress impairs postnatal oligodendrogenesis and adult emotional behaviour through activitydependent mechanisms. Mol Psychiatry. https://doi.org/10.1038/ s41380-019-0493-2 (2019).

5. Zhang Y, et al. CircDYM ameliorates depressive-like behavior by targeting miR-9 to regulate microglial activation via HSP90 ubiquitination. Mol Psychiatry. https://doi.org/10.1038/s41380-0180285-0 (2018).

6. Panda AC. Circular RNAs act as miRNA sponges. Adv Exp Med Biol. 2018;1087:67-79. https://doi.org/10.1007/978-981-131426-1_6

7. Tan A, et al. Effects of the KCNQ channel opener ezogabine on functional connectivity of the ventral striatum and clinical symptoms in patients with major depressive disorder. Mol Psychiatry. https://doi.org/10.1038/s41380-018-0283-2 (2018).

8. Kin K, et al. Cell encapsulation enhances antidepressant effect of the mesenchymal stem cells and counteracts depressive-like behavior of treatment-resistant depressed rats. Mol Psychiatry. https://doi.org/10.1038/s41380-018-0208-0 (2018).

9. Orrico-Sanchez A, et al. Antidepressant efficacy of a selective organic cation transporter blocker in a mouse model of depression. Mol Psychiatry. https://doi.org/10.1038/s41380-019-0548-4 (2019).

10. Han Y, et al. Systemic immunization with altered myelin basic protein peptide produces sustained antidepressant-like effects. Mol Psychiatry. https://doi.org/10.1038/s41380-019-0470-9 (2019).

11. Wittenberg GM, et al. Effects of immunomodulatory drugs on depressive symptoms: A mega-analysis of randomized, placebocontrolled clinical trials in inflammatory disorders. Mol Psychiatry. https://doi.org/10.1038/s41380-019-0471-8 (2019).

12. Beydoun, MA, et al. Systemic inflammation is associated with depressive symptoms differentially by sex and race: a longitudinal study of urban adults. Mol Psychiatry. https://doi.org/10.1038/ s41380-019-0408-2 (2019).

13. Felger, JC, et al. What does plasma CRP tell us about peripheral and central inflammation in depression? Mol Psychiatry. https:// doi.org/10.1038/s41380-018-0096-3 (2018).

14. Clark, SL, et al. A methylation study of long-term depression risk. Mol Psychiatry. https://doi.org/10.1038/s41380-019-0516-z (2019).

15. Aberg, KA, et al. Methylome-wide association findings for major depressive disorder overlap in blood and brain and replicate in independent brain samples. Mol Psychiatry. https://doi.org/10. 1038/s41380-018-0247-6 (2018).

16. Wei, YB, et al. A functional variant in the serotonin receptor 7 gene (HTR7), rs7905446, is associated with good response to SSRIs in bipolar and unipolar depression. Mol Psychiatry. https:// doi.org/10.1038/s41380-019-0397-1 (2019).

17. Chottekalapanda, R, et al. AP-1 controls the p11-dependent antidepressant response. Mol Psychiatry. https://doi.org/10.1038/ s41380-020-0767-8 (2020). 